Canadian

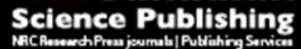

Canadian Journal of Physiology and Pharmacology Revue canadienne de physiologie et pharmacologie

The ergogenic supplement $\beta$-hydroxy- $\beta$-methylbutyrate (HMB) attenuates insulin resistance through suppressing GLUT-2 in rat liver

\begin{tabular}{|r|l|}
\hline Journal: & Canadian Journal of Physiology and Pharmacology \\
\hline Manuscript ID & cjpp-2015-0385.R1 \\
\hline Manuscript Type: & Article \\
\hline Date Submitted by the Author: & 14 -Oct-2015 \\
\hline Complete List of Authors: & $\begin{array}{l}\text { Sharawy, Maha ; Faculty of Pharmacy, Mansoura University, Department of } \\
\text { Pharmacology and Toxicology } \\
\text { El-Awady, Mohammed; Faculty of Pharmacy, Pharmacology \& Toxicology } \\
\text { Megahed, Nirmeen; Faculty of Medicine, Mansoura University, Department } \\
\text { of Pathology } \\
\text { Gameil, Nariman; Faculty of Pharmacy, Mansoura University, Department } \\
\text { of Pharmacology and Toxicology }\end{array}$ \\
\hline Keyword: & \multicolumn{2}{|c}{} \\
\hline
\end{tabular}




\title{
The ergogenic supplement $\beta$-hydroxy- $\beta$-methylbutyrate (HMB) attenuates insulin resistance through suppressing GLUT-2 in rat liver
}

\author{
Maha H. Sharawy ${ }^{1}$, Mohammed S. El-Awady ${ }^{1 *}$, Nirmeen Megahed ${ }^{2}$, Nariman M. \\ Gameil $^{1}$ \\ ${ }^{1}$ Department of Pharmacology and Toxicology, Faculty of Pharmacy, Mansoura \\ University, Mansoura, 35516, Egypt \\ ${ }^{2}$ Department of Pathology, Faculty of Medicine, Mansoura University, Mansoura, \\ 35516, Egypt
}

*Corresponding author:

Mohammed S. El-Awady, PhD

Department of Pharmacology and Toxicology,

Faculty of Pharmacy, Mansoura University, Mansoura 35516, Egypt

Tel. 0020502247496

Fax. 0020502247496

E-mail: mohamshh@yahoo.com 


\begin{abstract}
This study investigates the effect of the ergogenic supplement $\beta$-hydroxy- $\beta$ methylbutyrate (HMB) on insulin resistance induced by high fructose diet (HFD) in rats. Male Sprague Dawley rats were fed 60\% HFD for 12 weeks and HMB (320 $\mathrm{mg} / \mathrm{kg} /$ day, orally) for 4 weeks. HFD significantly increased fasting insulin, fasting glucose, glycosylated hemoglobin $\left(\mathrm{Hb}_{\mathrm{Alc}}\right)$, liver glycogen content and homeostasis model assessment of insulin resistance (HOMA-IR) index, while it decreased glucose and insulin tolerance. Furthermore, HFD significantly increased serum triglycerides (TG), low density lipoprotein cholesterol (LDL-C) and very low density lipoprotein cholesterol (VLDL-C) levels, while it significantly decreased high density lipoprotein cholesterol (HDL-C). Moreover, HFD significantly increased mRNA expression of glucose transporter type-2 (GLUT-2), the mammalian target of rapamycin (mTOR) and sterol regulatory element-binding protein-1c (SREBP-1c) but decreased peroxisome proliferator-activated receptor-alpha $(\operatorname{PPAR}-\alpha)$ in liver. Aortic relaxation to acetylcholine (ACh) was impaired and histopathology showed severe hepatic steatosis. HMB significantly increased insulin tolerance and decreased fasting insulin, HOMA-IR, $\mathrm{Hb}_{\mathrm{Al}}$, hepatic glycogen content, serum TG, LDL-C and VLDL-C. Additionally, HMB enhanced ACh-induced relaxation, ameliorated hepatic steatosis and decreased mRNA expression of GLUT-2. In conclusion: HMB may attenuate insulin resistance and hepatic steatosis through inhibiting GLUT-2 in liver.
\end{abstract}

\title{
Keywords
}

Insulin resistance- $\beta$-hydroxy- $\beta$-methylbutyrate - High fructose diet - Glucose transporter type-2 - Hepatic steatosis 


\begin{abstract}
Abbreviations
HMB: $\beta$-hydroxy- $\beta$-methylbutyrate; HFD: high fructose diet; GLUT-2: glucose transporter type-2; mTOR: the mammalian target of rapamycin; SREBP-1c: sterol regulatory element-binding protein- $1 \mathrm{c}$ and PPAR- $\alpha$ : peroxisome proliferator-activated receptor-alpha.
\end{abstract}

\title{
Introduction
}

Body builders and athletes use ergogenic supplements extensively to maintain their strength and increase their muscle mass and exercise performance. Many of these supplements have hazardous effects on health if taken without any considerations of the athlete's medical condition or without consulting a physician. One of the most widely used supplements is $\beta$-hydroxy- $\beta$-methylbutyrate (HMB) (Molfino et al. 2013).

HMB is a metabolite of the essential branched chain amino acid leucine (Van and Nissen 1992). HMB supplementation has been found to be useful for weight training athletes, the elderly, or in patients with diseases associated with muscle wasting such as cancer and AIDS (Pinheiro et al. 2013). Supplemental HMB is usually taken at a daily dose of 3 grams/day which was found to be safe (Nissen et al. 2000).

The role of HMB in glucose and insulin homeostasis is controversial. Few reports have emerged showing glucose intolerance and elevation in insulin level after HMB supplementation. For example, glucose intolerance induced by glucocorticoid in Wistar rats was further impaired by co-administration of HMB, while HMB alone did not cause insulin resistance (Nunes et al. 2013). This effect was attributed mainly to glucocorticoid effect on lipolysis of adipose tissue (Geer et al. 2014). In addition, HMB

supplementation for 4 weeks in healthy sedentary Wistar rats reduced glucose tolerance and elevated insulin (Yonamine et al. 2014). Moreover, HMB supplementation slightly 
increased insulin level after exercise in humans (Townsend et al. 2015). However, the exact mechanism linking HMB to insulin resistance is still unclear.

Insulin resistance is a condition in which liver, skeletal muscle and adipose tissue fail to respond to the circulating levels of insulin (Virkamaki et al. 1999). It is associated with obesity, hyperinsulinemia, hyperlipidemia, fatty liver and inflammation (Ye 2013).

There are many experimental models to induce insulin resistance among which the use of glucocorticoid, sodium nitrite, high fat diet or high fructose diet (HFD) (AlGayyar et al. 2015;Deer et al. 2015;Su et al. 2014). The HFD model is widely used because it mimics the insulin resistance found in human (Bezerra et al. 2000). It was previously reported that even moderate fructose consumption alters both insulin sensitivity and lipid metabolism (Aeberli et al. 2013). Fructose, a lipogenic monosaccharide, is considered a contributor to nearly all of the manifestations of the insulin resistance syndrome (Elliott et al. 2002).

Since the relation between HMB supplementation and insulin resistance remains controversial, therefore, the present study aimed to investigate the effect of HMB on insulin resistance and the possible underlying mechanisms.

\section{Materials and Methods}

\section{Drugs and chemicals}

Fructose was purchased from Safety Misr (Cairo, Egypt); HMB (Calcium salt) from Optimum Nutrition (Sunrise, FL, USA); anthrone from Alpha Chemika (Mumbai, India). Acetylcholine hydrochloride ( $\mathrm{ACh}$ ); pentobarbital sodium; phenylephrine hydrochloride (PE) and sodium nitroprusside (SNP) were purchased from Sigma Aldrich chemical Co. (St. Louis, MO, USA). 


\section{Experimental animals}

Male Sprauge Dawely rats with average age of 6-8 weeks were purchased from "Egyptian Organization for Biological Products and Vaccines", Giza, Egypt. The study protocol followed the ethical guidelines for the care and use of laboratory animals of the Ethical Committee of the Faculty of Pharmacy, Mansoura University, Egypt, which is in accordance with the Principles of Laboratory Animal Care (NIH publication no. 85-23, revised 1985).

\section{Experimental protocol}

The rats were randomly divided into four groups, each of 8 rats. Group (1): Control group: Rats receiving regular chow for 12 weeks. Group (2): HFD group: Rats receiving 60\% HFD for 12 weeks (Liao et al. 2010). Group (3): HMB group: Rats receiving regular chow for 12 weeks and HMB (320 mg/kg/day, orally) using a fine gastric gavage directly introduced to the stomach from week 9 to 12 (Pinheiro et al. 2012). Group (4): HFD+HMB group: Rats receiving 60\% HFD for 12 weeks and HMB (320 mg/kg/day, orally) from week 9 to 12 .

The composition of regular chow was $60 \%$ carbohydrates, $20 \%$ protein, $4 \%$ fats, $2 \%$ fibers, $14 \%$ minerals and vitamins. In HFD, fructose was added instead of carbohydrates. All rats had free access to drinking water and chow for 12 weeks. The weight of rats was recorded weekly and the daily food intake was calculated.

\section{Oral Glucose Tolerance Test (OGTT)}

Forty-eight hours prior to the end day, animals were fasted for 12 hours and $40 \%$ glucose solution ( $2 \mathrm{~g} / \mathrm{kg}$ body weight) was given orally by gastric tube to conscious rats. Blood glucose level was measured at 0,30,60, 120 and 180 minutes after glucose administration. The serum blood glucose level was measured using a glucometer using 
glucose dehydrogenase method (Glucodoctor auto, All Medicus co., Gyeonggi-do, Republic of Korea) after pricking the tail vein.

\section{Insulin Tolerance Test (ITT)}

Twenty-four hours before the end day, animals were fasted for 12 hours and a dose of $0.5 \mathrm{IU} / \mathrm{kg}$ human soluble insulin (Actrapid, Novo Nordisk, Bagsvaerd, Denmark) was injected subcutaneously to conscious rats. Blood glucose level was determined at $0,30,60,120$ and $180 \mathrm{~min}$ after insulin administration. The serum blood glucose level was calculated using a small blood drop from the tail.

\section{Sample preparation}

At the end of 12 weeks, the rats were fasted for 12 hours and euthanized using pentobarbital sodium (60 mg/kg, i.p.) and blood samples were collected to prepare serum and heparinized plasma. Liver tissues were immediately collected and snapfrozen in liquid nitrogen, stored at $-80^{\circ} \mathrm{C}$ to be used later for real-time RT-PCR, and glycogen content determination. Another lobe of liver was fixed in $10 \%$ buffered formalin for subsequent histopathological examination. In addition, thoracic aortic rings were isolated for in vitro vascular reactivity measurement.

\section{Determination of fasting serum glucose, insulin and insulin resistance index}

The fasting glucose level was measured in serum using an enzymatic colorimetric commercial kit purchased from Biodiagnostic Company (Giza, Egypt). The fasting rat insulin was measured in serum using a rat insulin ELISA kit (Mercodia Developing Diagnostics, Uppsala, Sweden).

Homeostasis model assessment of insulin resistance (HOMA-IR) index was calculated using HOMA2 Calculator, developed by the Diabetes Trial Unit, University of Oxford, Oxford. UK (Levy et al. 1998). 


\section{Estimation of glycosylated hemoglobin (HbA1c)}

Glycosylated hemoglobin was determined in whole blood using commercial kit containing cation-exchange resin, according to the manufacturer's instructions (Stanbio Laboratories, TX, USA).

\section{Estimation of liver glycogen content}

Liver glycogen content was assessed using the method described by (Seifter et al. 1950). The method is based on the digestion of tissue in $30 \% \mathrm{KOH}$ and precipitating quantitative yield of glycogen with $95 \%$ ethanol and saturated sodium sulphate solution. The glycogen content was estimated by the use of anthrone reagent dissolved in sulphuric acid that causes dehydration of carbohydrates that condenses in the presence of anthrone to produce bluish green color measured at $620 \mathrm{~nm}$ and glycogen was expressed as $\mathrm{mg} / 100 \mathrm{~g}$ tissue.

\section{Measurement of lipid profile in serum}

Serum triglycerides (TG) and total cholesterol (TC) were estimated in serum using commercial kits (Spinreact, Santa Coloma, Spain) according to manufacturer's instructions. High density lipoprotein cholesterol (HDL-C) was estimated in serum using commercial kit (Vitro Scient., Hannover, Germany). Low density lipoprotein cholesterol (LDL-C) and very low density lipoprotein cholesterol (VLDL-C) were determined according to the following equations: VLDL-C $=\mathrm{TG} / 5$ while $\mathrm{LDL}-\mathrm{C}=\mathrm{TC}$ [VLDL-C + HDL-C] (Yadav et al. 2007).

\section{Determination of in vitro vascular reactivity}

The descending thoracic aorta was isolated and rapidly placed in cold oxygenated physiological salt solution of the following composition (mmol/L): $\mathrm{NaCl}$ $118, \mathrm{KCl} 4.7, \mathrm{CaCl}_{2} 2.5$, and $\mathrm{MgSO}_{4} \cdot 7 \mathrm{H}_{2} \mathrm{O} 1.2, \mathrm{KH}_{2} \mathrm{PO}_{4} 1.2$, glucose 11.1 and $\mathrm{NaHCO}_{3}$ 
25; $\mathrm{pH} 7.4$, then the aorta was dissected free of fat and connective tissue, and cut into rings $(2-4 \mathrm{~mm})$ each.

Each aortic ring was mounted between two stainless steel hooks in a $10 \mathrm{ml}$ organ bath at $37^{\circ} \mathrm{C}$ and was filled with physiological salt solution which was aerated with a mixture of $95 \% \mathrm{O}_{2}$ and $5 \% \mathrm{CO}_{2}$. The aortic ring was allowed to equilibrate under $1 \mathrm{~g}$ of force for 60 minutes during which the physiological solution was changed every 15 minutes. Vascular reactivity was measured using Riegestab K30 force transducer (Hugo Sachs electronic, D7806 march, Germany), and recorded with a Powerlab unit/400 with Chart v4.2 software (ADInstruments Pty Ltd., Australia). After equilibration, the vascular responsiveness was evaluated by three times stimulation with $80 \mathrm{mM} \mathrm{KCl}$ until a reproducible contractile response was obtained.

Vascular reactivity to PE $\left(10^{-10}-10^{-5} \mathrm{M}\right)$ was determined by constructing cumulative concentration response curve. Additionally, the aortic ring was precontracted with $1 \mu \mathrm{M}$ PE and vascular relaxation to ACh $\left(10^{-10}-10^{-5} \mathrm{M}\right)$ and SNP $\left(10^{-10}-10^{-5} \mathrm{M}\right)$ were measured.

\section{Measurement of $m R N A$ expression of GLUT-2, mTOR, SREBP-1c and PPAR- $\alpha$ using real-time RT-PCR}

RNA was isolated from liver samples using TRIZOL reagent (Ambion, Carlsbad, CA, USA) according to manufacturer's instructions. First strand cDNA was synthesized from $2 \mu \mathrm{g}$ of total RNA in $20 \mu \mathrm{l}$ volume using Revert Aid First Strand cDNA Synthesis Kit (Thermo Scientific, Rockford, IL, USA) according to the manufacturer's protocol. Real-time PCR was performed in Rotor Gene Q thermocycler (Qiagen, Hilden, Germany), using HOT Firepol Evagreen qPCR mix plus kit (Solis BioDyne, Tartu, Estonia).

The mRNA levels of glucose transporter type 2 (GLUT-2), the mammalian target of rapamycin (mTOR), sterol regulatory element-binding protein-1c (SREBP-1c) 
and peroxisome proliferator-activated receptor-alpha (PPAR- $\alpha$ ) were measured in duplicates and normalized relative to $18 \mathrm{~S}$ ribosomal RNA (Rn18S) in the same sample. Sequences of primers for the GLUT-2, mTOR, SREBP-1c, PPAR- $\alpha$ and Rn18S (Invitrogen by Life Technologies, Carlsbad, CA, USA), were as the following:

GLUT-2: sense: TGACTGGAGCCCTCTTGATG, antisense:

CCCCTGAGTGTGGTTGGAG, amplicon size =151; mTOR: sense:

TATGACCCCAACCAGCCAAT, antisense: AGGAAAACAAACTCGTGCCC, amplicon size =125; SREBP-1c: sense: CTGTAGGTCACCGTTTCTTCG, antisense: CAGTTCAATGCTCGCTCCAG, amplicon size =151; PPAR-a: sense:

AATCCACGAAGCCTACCTGA, antisense: CCTCTGCCTCCTTGTTTTCA, amplicon size $=182$ and Rn18S: sense: AGTTGGTGGAGCGATTTGTC, antisense:

GAACGCCACTTGTCCCTCTA, amplicon size $=121$.

The results were expressed as an $n$-fold change of the relative expression levels of target genes from control group using $\Delta \Delta \mathrm{C}_{\mathrm{t}}$ method.

\section{Histopathological examination:}

The $10 \%$ buffered formalin fixed liver tissue was embedded in paraffin wax, sectioned $(6 \mu \mathrm{m})$ and stained with hematoxylin-eosin (H\&E) and samples were assessed for the presence of steatosis microscopically (Leica Imaging Systems, Cambridge, UK). The hepatic steatosis was evaluated by semiquantitative scale from 0 to $3 ; 0=$ no steatosis, $1=$ mild steatosis less than 33\%, $2=$ moderate steatosis more than $33 \%$ and less than $66 \%$ and $3=$ severe steatosis more than $66 \%$. The pathologist performing histopathological evaluation was blinded to the study treatment assignment.

\section{Statistical analysis:}

Data are expressed as mean \pm standard error of mean (SEM). Statistical analysis and graphing were carried out using GraphPad software Prism V 5.02 (GraphPad 
Software Inc., San Diego, CA, USA). Vascular relaxation was calculated as percentage of the maximal contraction induced by $1 \mu \mathrm{M}$ PE. The highest response obtained was considered as the maximum response $\left(\mathrm{E}_{\max }\right)$ and $\mathrm{pEC}_{50}$ (negative log of the concentration producing $50 \%$ of maximal response) was determined from non-linear regression analysis (four-parameter curve fit). One-way analysis of variance (ANOVA) followed by Tukey-Kramer's multiple comparisons post-hoc test were used to measure significant differences between groups. Statistical significance was considered at $\mathrm{p}<0.05$. Histopathological steatosis scoring was compared using Kruskal-Wallis followed by Dunn's multiple comparison test.

\section{Results}

Effect of HMB on HFD-induced changes in body weight gain and mean daily food intake in rats

The body weight gain and mean food intake were not significantly altered among groups after 12 weeks (Table 1).

\section{Effect of HMB on HFD-induced changes in OGTT and ITT}

The HFD caused a significant $(p<0.05, n=8)$ increase in area under the curve (AUC) of blood glucose level in OGTT by 113\% compared to the control group (Figure 1). HMB treatment did not significantly reduce HFD-induced increase in AUC.

Insulin sensitivity measured by ITT was significantly $(p<0.05, n=8)$ impaired by the HFD as shown by the increase in AUC which changed to $159 \%$ compared to the control group. HMB treatment in HFD rats decreased AUC significantly $(p<0.05, n=8)$ (Figure 1) by $19 \%$ compared to HFD group. HMB treatment had no significant effect on OGTT or ITT in control rats. 


\section{Effect of HMB on HFD-induced changes in fasting serum glucose, insulin, HOMA- IR and $\mathrm{Hb} A_{1 C}$}

The HFD caused a significant state of insulin resistance indicated by a significant $(p<0.05, n=8)$ increase in fasting insulin level and HOMA-IR index by 344 $\%$ and $362 \%$ respectively compared to the control group (Table 1). Moreover, HFD caused a significant state of hyperglycemia as shown by the increase in fasting glucose level and $\mathrm{HbA}_{1 \mathrm{C}}$ by $109 \%$ and $137 \%$ respectively compared to the control group.

Treatment with HMB decreased the level of insulin resistance induced by HFD by significantly $(p<0.05, n=8)$ decreasing both fasting insulin level and HOMA-IR index which decreased by $22 \%$ and $27 \%$ respectively compared to HFD group. It also decreased $\mathrm{HbA}_{1 \mathrm{C}}$ levels by $27 \%$ compared to HFD group. HMB did not significantly affect fasting glucose (Table 1). HMB treatment had no significant effect on fasting glucose, insulin, HOMA-IR or $\mathrm{HbA}_{1 \mathrm{c}}$ in control animals.

\section{Effect of HMB on HFD-induced changes in liver glycogen content}

Liver glycogen content was significantly $(p<0.05, n=8)$ elevated by HFD by 207 $\%$ compared to the control group (Figure 1).

Treatment with HMB in HFD rats from week 9 to 12 managed to decrease liver glycogen content significantly $(p<0.05, n=8)$, which was reduced by $43 \%$ compared HFD group. HMB treatment had no significant effect on liver glycogen content in control animals.

\section{Effect of HMB on HFD-induced changes in lipid profile in serum}

The HFD caused a significant $(p<0.05, n=8)$ increase in TG, LDL-C and VLDL-C levels by $318 \%, 638 \%$ and $297 \%$ respectively (Figure 2) and it caused a significant decrease in HDL-C level by $19 \%$ compared to the control group, while TC level wasn't significantly affected by HFD. 
Treatment with HMB significantly $(p<0.05, n=8)$ decreased the HFD-induced elevated levels of TG, LDL-C and VLDL-C levels, which was decreased by $67 \%, 72$ $\%$ and $64 \%$ respectively compared to HFD group, while, it did not attenuate the decreased level of HDL-C. HMB treatment had no significant effect on lipid profile in control rats.

\section{Effect of HMB on HFD-induced changes in vascular reactivity in vitro}

The HFD had no significant effect on $\mathrm{KCl}$ or PE-induced contraction or SNPinduced relaxation in aortic rings (Figure 3) when compared to control group. On the other hand, HFD caused a significant $(p<0.05, n=6)$ decrease in endothelium-dependent ACh-induced maximal relaxation, where the $\left(\mathrm{E}_{\max }\right)$ decreased significantly by $57 \%$ compared to control group, however, it had no significant effect on $\mathrm{pEC}_{50}$.

HMB in HFD rats significantly $(p<0.05, n=6)$ enhanced vascular reactivity by increasing ACh-induced maximal relaxation $\left(\mathrm{E}_{\max }\right)$, which increased by $205 \%$ compared to the HFD group. HMB treatment had no significant effect on vascular reactivity in control rats.

Effect of HMB on HFD-induced changes in mRNA expression of GLUT-2, mTOR, SREBP-1c and PPAR- $\alpha$

The HFD caused a significant $(p<0.05, n=6)$ elevation in mRNA expression of GLUT-2, mTOR and SREBP-1c by $242 \%, 359 \%$ and $678 \%$ respectively, and a significant decrease in PPAR- $\alpha$ mRNA expression by $68 \%$ when compared to control group (Figure 4).

Treatment with HMB significantly $(p<0.05, n=6)$ decreased GLUT-2 expression by $36 \%$ compared to HFD group, but it did not significantly prevent HFD-induced changes in mRNA expression of mTOR, SREBP-1c and PPAR- $\alpha$. HMB treatment had 
no significant effect on mRNA expression of GLUT-2, mTOR, SREBP-1c and PPAR- $\alpha$ in normal rats.

\section{Effect of HMB on HFD-induced changes in histopathology}

Liver specimen $(n=6)$ of control group showed normal hepatic architecture with normal hepatocytes arranged in 1-2 thickness plates with no evidence of steatosis, with mean score $=0.33$ (Figure 5A), while HFD rats showed severe steatosis affecting $>66 \%$ of hepatocytes mainly periportal (microvesicular and macrovesicular steatosis), with mean score $=2.67$ (Figure 5B). Treatment with HMB in HFD rats showed minimal steatosis (15\% of hepatocytes) showing both microvesicular and macrovesicular steatosis, with mean score $=1$ (Figure 5D). HMB treatment in normal rats showed normal hepatocytes radiating from central veins with no evidence of steatosis, with mean score $=0.33$ (Figure 5C).

\section{Discussion}

In this study, feeding rats with HFD for 12 weeks showed a decline in insulin sensitivity which was shown by the impaired insulin and glucose tolerance. The state of insulin resistance was confirmed by elevated levels of fasting insulin, fasting glucose, $\mathrm{HbA}_{1 \mathrm{C}}$ and HOMA-IR. Furthermore, liver glycogen content was increased and dyslipidemia was induced. HFD managed to induce an endothelial dysfunction, which was observed in the impairment of endothelial-dependent relaxation in isolated aortic rings. Additionally, the mRNA expression of GLUT-2, mTOR and SREBP-1c was increased while that of PPAR- $\alpha$ was decreased.

Starting HMB treatment remarkably alleviated HFD-induced insulin resistance as shown by the increase in insulin tolerance and the decrease in fasting insulin, HOMA-IR index and $\mathrm{Hb}_{\mathrm{Alc}}$. In addition, $\mathrm{HMB}$ treatment decreased liver glycogen 
content and decreased the elevated levels of TG, VLDL-C and LDL-C. Moreover, endothelial-dependent relaxation was improved. Additionally, the elevated mRNA expression of GLUT-2 was decreased and hepatic steatosis was attenuated.

The duration of HFD feeding varies mostly from 4 to 12 weeks to induce insulin resistance (Liao et al. 2010; Shawky et al. 2014;Su et al. 2009). In this study, by the end of 8 weeks of HFD feeding insulin resistance was confirmed by the elevated levels of fasting glucose and insulin and the alteration of lipid profile. In this study, insulin resistance was elucidated by the hyperinsulinemia, hyperglycemia, and elevation in HOMA-IR, $\mathrm{HbA}_{1 \mathrm{c}}$, and liver glycogen content in addition to decrease in insulin and glucose tolerance. These findings were in accordance with previous studies (Madani et al. 2012;Reddy et al. 2009; Shawky et al. 2014; Yadav et al. 2007; Yadav et al. 2009).

Fructose metabolism in the liver mainly forms glycogen and triglycerides; the effect of fructose is inhibitory on glycogen breakdown and stimulatory on its synthesis (Koo et al. 2008; Mayes 1993). Elevated level of $\mathrm{HbA}_{1 \mathrm{C}}$ correlates with increased fasting blood glucose level in insulin resistance model.

In the present study, HMB treatment managed to decrease liver glycogen content and the slightly elevated $\mathrm{HbA}_{1 \mathrm{c}}$. In addition, GLUT-2 was measured as a major contributor to glucose and fructose homeostasis in the liver (Burcelin et al. 2000; Manolescu et al. 2007; Wood and Trayhurn 2003). GLUT-2 is the main fructose transporter in the hepatocytes where most of the ingested fructose is metabolized. It plays an important role in the metabolic disorders associated with fructose consumption (Douard and Ferraris 2013). GLUT-2 may be involved in insulin resistance where its expression increases in liver (Liu et al. 2015; Mathur et al. 2015; Narasimhan et al. 2015). In the present study, GLUT-2 mRNA expression was reduced by HMB treatment. This could be one of the main mechanisms involved in HMB beneficial 
effects on insulin resistance in HFD model through decreasing the amount of transported fructose to the liver.

In addition to the impairment of glucose homeostasis in HFD model, hepatic steatosis due to excessive triglycerides level was elucidated in this study. HFD caused dyslipidemia, which was shown especially by the elevation of TG. HMB treatment attenuated both dyslipidemia and hepatic steatosis. A previous study showed that HMB decreased LDL-C in human (Nissen et al. 2000). However, no previous studies measured HMB effect on hypertriglyceridemia and hepatic steatosis in HFD model of insulin resistance.

In this study, HFD caused an increase in mRNA expression of SREBP-1c in the liver, while it decreased mRNA expression of PPAR- $\alpha$ in the liver. These findings were consistent with previous studies (Basciano et al. 2005; Matsuzaka et al. 2004;Nagai et al. 2002; Shiomi et al. 2015). SREBP-1c regulates many lipogenic enzymes as $\beta$-hydroxy$\beta$-methylglutaryl coenzyme A (HMG-CoA) and fatty acid synthase (FAS) that are key enymes in de novo lipogenesis (Matsuzaka et al. 2004). Additionally, fatty acid $\beta$ oxidation and ketogenesis in the liver are controlled by PPAR- $\alpha$ (Lefebvre et al. 2006), which is impaired in HFD model (Nagai et al. 2002). These results could explain lipid accumulation and hepatic steatosis as seen in histopathological examination of this study. However, HMB treatment did not attenuate the elevation in SREBP-1c or the decrease in PPAR- $\alpha$ mRNA expression

Fructose can bypass the rate-limiting reaction of phosphofructokinase (Mayes 1993) serving as unregulated source of both glycerol-3-phosphate and acetyl-CoA, which are used by the liver for the production of free fatty acids (FFA) (Basciano et al. 2005). The increase in FFA production enhances TG biosynthesis. Since HMB decreased the mRNA expression of GLUT-2 leading to decreasing fructose entrance 
into hepatic cells as a major precursor for lipogenesis in our model. Therefore, HMB may attenuate dyslipidemia and hepatic steatosis through its effect on GLUT-2.

Insulin signaling is inhibited by the intermediates produced during TG synthesis as mTOR, protein kinase-C (PKC) and ceramide (Nagle et al. 2009). PKC and mTOR affect the phosphorylation of insulin receptor substrate-1 (IRS-1) that is accompanied by an impaired association between IRS-1 and PI3K (Gual et al. 2005), preventing PI3K activation (Li et al. 1999; Moeschel et al. 2004). Ceramide inhibits Akt-mediated insulin signaling resulting in insulin resistance.

In this study treatment with HMB for 4 weeks reduced insulin resistance, HOMA-IR index and increased insulin tolerance, without affecting mTOR mRNA expression. However, previous study has shown that mTOR expression in skeletal muscle increases after HMB consumption in a model of skeletal muscle hypertrophy in rats, this discrepancy may be due to the difference in the model and the difference in mTOR expression in skeletal muscle than in liver (Pimentel et al. 2011).

HMB was previously shown to stimulate PI3K/Akt pathway involved in insulin signaling (Kornasio et al. 2009). This effect of HMB may be related to its attenuating effect on dyslipidemia, where decreasing TG intermediates will subsequently enhance insulin signaling.

Insulin resistance is also characterized by impaired endothelial-dependent relaxation (Takagawa et al. 2001). The PI3K/Akt pathway is compromised in fructoseinduced insulin resistance model leading to a decrease in endothelial nitric oxide synthase (eNOS) phosphorylation and a decrease in nitric oxide (NO) release. The imbalance between the decreased NO release and secretion of endothelin-1 (which has vasoconstrictor actions related to insulin) can be the cause of endothelial dysfunction in insulin resistance (Muniyappa and Sowers 2013). Endothelial dysfunction is widely reported in fructose-induced insulin resistance model (Shawky et al. 2014; Vasudevan et 
al. 2006). In this study, HMB treatment alleviated endothelial dysfunction induced by HFD. This protective effect of HMB may be due to its anti-inflammatory effect through inhibiting tumor necrosis factor- $\alpha(\mathrm{TNF}-\alpha)$ and nuclear factor kappa-B (Kraemer et al. 2014; Nunes et al. 2008; Nunes et al. 2011). Additionally, the PI3K/Akt pathway may be improved by HMB (Kornasio et al. 2009) leading to enhanced endothelial function.

\section{In Conclusion:}

HMB may attenuate insulin resistance and hepatic steatosis through inhibiting GLUT-2 in liver without affecting SREBP-1c, mTOR or PPAR- $\alpha$. Therefore, the use of HMB supplementation in insulin resistance may have beneficial effect.

\section{Acknowledgment:}

The authors acknowledge Dr. Mahmoud Gabr and the staff members of the Biotechnology Department, Urology and Nephrology, Center, Mansoura University, Egypt, for kindly sharing the facilities of their laboratory and their helpful support.

\section{Conflict of interest statement:}

None declared.

\section{References}

Aeberli, I., Hochuli, M., Gerber, P.A., Sze, L., Murer, S.B., Tappy, L., Spinas, G.A., and Berneis, K. 2013. Moderate amounts of fructose consumption impair insulin sensitivity in healthy young men: a randomized controlled trial. Diabetes Care, 36: 15015.6 
Al-Gayyar, M.M., Alyoussef, A., Hamdan, A.M., Abbas, A., Darweish, M.M., and ElHawwary, A.A. 2015. Cod liver oil ameliorates sodium nitrite-induced insulin resistance and degradation of rat hepatic glycogen through inhibition of cAMP/PKA pathway. Life Sci. 120: 13-21.

Basciano, H., Federico, L., and Adeli, K. 2005. Fructose, insulin resistance, and metabolic dyslipidemia. Nutr. Metab. (Lond.) 2: 5.

Bezerra, R.M., Ueno, M., Silva, M.S., Tavares, D.Q., Carvalho, C.R., and Saad, M.J. 2000. A high fructose diet affects the early steps of insulin action in muscle and liver of rats. J. Nutr. 130: 1531-1535.

Burcelin, R., Dolci, W., and Thorens, B. 2000. Glucose sensing by the hepatoportal sensor is GLUT2-dependent: in vivo analysis in GLUT2-null mice. Diabetes, 49: 16431648.

Deer, J., Koska, J., Ozias, M., and Reaven, P. 2015. Dietary models of insulin resistance. Metabolism, 64: 163-171.

Douard, V., and Ferraris, R.P. 2013. The role of fructose transporters in diseases linked to excessive fructose intake. J. Physiol. 591: 401-414.

Elliott, S.S., Keim, N.L., Stern, J.S., Teff, K., and Havel, P.J. 2002. Fructose, weight gain, and the insulin resistance syndrome. Am. J. Clin. Nutr. 76: 911-922.

Geer, E.B., Islam, J., and Buettner, C. 2014. Mechanisms of glucocorticoid-induced insulin resistance: focus on adipose tissue function and lipid metabolism. Endocrinol. Metab. Clin. North. Am. 43: 75-102.

Gual, P., Le Marchand-Brustel, Y., and Tanti, J.F. 2005. Positive and negative regulation of insulin signaling through IRS-1 phosphorylation. Biochimie, 87: 99-109.

Koo, H.Y., Wallig, M.A., Chung, B.H., Nara, T.Y., Cho, B.H., and Nakamura, M.T.

2008. Dietary fructose induces a wide range of genes with distinct shift in carbohydrate 
and lipid metabolism in fed and fasted rat liver. Biochim. Biophys. Acta. 1782: 341348.

Kornasio, R., Riederer, I., Butler-Browne, G., Mouly, V., Uni, Z., and Halevy, O. 2009. Beta-hydroxy-beta-methylbutyrate (HMB) stimulates myogenic cell proliferation, differentiation and survival via the MAPK/ERK and PI3K/Akt pathways. Biochim. Biophys. Acta. 1793: 755-763.

Kraemer, W.J., Hatfield, D.L., Comstock, B.A., Fragala, M.S., Davitt, P.M., Cortis, C., Wilson, J.M., Lee, E.C., Newton, R.U., Dunn-Lewis, C., Hakkinen, K., Szivak, T.K., Hooper, D.R., Flanagan, S.D., Looney, D.P., White, M.T., Volek, J.S., and Maresh, C.M. 2014. Influence of HMB supplementation and resistance training on cytokine responses to resistance exercise. J. Am. Coll. Nutr. 33: 247-255.

Lefebvre, P., Chinetti, G., Fruchart, J.C ,.and Staels, B. 2006. Sorting out the roles of PPAR alpha in energy metabolism and vascular homeostasis. J. Clin. Invest. 116: 571580.

Levy, J.C., Matthews, D.R., and Hermans, M.P. 1998. Correct homeostasis model assessment (HOMA) evaluation uses the computer program. Diabetes Care, 21: 21912192.

Li, J., DeFea, K., and Roth, R.A. 1999. Modulation of insulin receptor substrate-1 tyrosine phosphorylation by an Akt/phosphatidylinositol 3-kinase pathway. J. Biol. Chem. 274: 9351-9356.

Liao, Q., Shi, D.H., Zheng, W., Xu, X.J., and Yu, Y.H. 2010. Antiproliferation of cardamonin is involved in mTOR on aortic smooth muscle cells in high fructoseinduced insulin resistance rats. Eur. J. Pharmacol. 641: 179-186. 
Liu, Y., Song, A., Zang, S., Wang, C., Song, G., Li, X ,.Zhu, Y., Yu, X., Li, L., Wang, Y., and Duan, L. 2015. Jinlida reduces insulin resistance and ameliorates liver oxidative stress in high-fat fed rats. J. Ethnopharmacol. 162: 244-252.

Madani, Z., Louchami, K., Sener, A., Malaisse, W.J., and Ait, Y.D. 20 .12Dietary sardine protein lowers insulin resistance, leptin and TNF-alpha and beneficially affects adipose tissue oxidative stress in rats with fructose-induced metabolic syndrome. Int. J. Mol. Med. 29: 311-318.

Manolescu, A.R., Witkowska, K., Kinnaird ,A., Cessford, T., and Cheeseman, C. 2007. Facilitated hexose transporters: new perspectives on form and function. Physiology. (Bethesda. ), 22: 234-240.

Mathur, R., Dutta, S., Velpandian, T., and Mathur, S.R. 2015. Psidium guajava Linn. leaf extract affects hepatic glucose transporter- 2 to attenuate early onset of insulin resistance consequent to high fructose intake: An experimental study. Pharmacognosy. Res. 7: 166-175.

Matsuzaka, T., Shimano, H., Yahagi, N., Amemiya-Kudo, M., Okazaki, H., Tamura, Y,. Iizuka, Y., Ohashi, K., Tomita, S., Sekiya, M., Hasty, A., Nakagawa, Y., Sone, H., Toyoshima, H., Ishibashi, S., Osuga, J., and Yamada, N. 2004. Insulin-independent induction of sterol regulatory element-binding protein-1c expression in the livers of streptozotocin-treated mice. Diabetes, 53: 560-569.

Mayes, P.A. 1993. Intermediary metabolism of fructose. Am. J. Clin. Nutr. 58: 754S$765 \mathrm{~S}$.

Moeschel, K., Beck, A., Weigert, C., Lammers, R., Kalbacher, H., Voelter, W., Schleicher, E.D., Haring, H.U., and Lehmann, R. 2004. Protein kinase C-zeta-induced phosphorylation of Ser318 in insulin receptor substrate-1 (IRS-1) attenuates the 
interaction with the insulin receptor and the tyrosine phosphorylation of IRS-1. J. Biol. Chem. 279: 25157-25163.

Molfino, A., Gioia, G., Rossi, F.F., and Muscaritoli, M. 2013. Beta-hydroxy-betamethylbutyrate supplementation in health and disease: a systematic review of randomized trials. Amino. Acids, 45: 1273-1292.

Muniyappa, R. and Sowers, J.R. 2013. Role of insulin resistance in endothelial dysfunction. Rev. Endocr. Metab. Disord. 14: 5-12.

Nagai, Y., Nishio, Y., Nakamura, T., Maegawa, H., Kikkawa, R., and Kashiwagi, A. 2002. Amelioration of high fructose-induced metabolic derangements by activation of PPARalpha. Am. J. Physiol. Endocrinol. Metab. 282: E1180-E1190.

Nagle, C.A., Klett, E.L., and Coleman, R.A. 2009. Hepatic triacylglycerol accumulation and insulin resistance. J. Lipid Res. 50 Suppl: S74-S79.

Narasimhan, A., Chinnaiyan, M., and Karundevi, B. 2015. Ferulic acid regulates hepatic GLUT2 gene expression in high fat and fructose-induced type-2 diabetic adult male rat. Eur. J. Pharmacol. 761: 391-397.

Nissen, S., Sharp, R.L., Panton, L., Vukovich, M., Trappe, S., and Fuller, J.C., Jr. 2000. beta-hydroxy-beta-methylbutyrate (HMB) supplementation in humans is safe and may decrease cardiovascular risk factors. J. Nutr. 130: 1937-1945.

Nunes, E.A., Goncalves-Neto, L.M., Ferreira, F.B., Dos, S.C., Fernandes, L.C., Boschero, A.C., Calder, P.C., and Rafacho, A. 2013. Glucose intolerance induced by glucocorticoid excess is further impaired by co-administration with beta-hydroxy-betamethylbutyrate in rats. Appl. Physiol. Nutr. Metab. 38: 1137-1146.

Nunes, E.A., Kuczera, D., Brito, G.A., Bonatto, S.J., Yamazaki, R.K., Tanhoffer, R.A ,. Mund, R.C., Kryczyk, M., and Fernandes, L.C. 2008. Beta-hydroxy-betamethylbutyrate supplementation reduces tumor growth and tumor cell proliferation ex 
vivo and prevents cachexia in Walker 256 tumor-bearing rats by modifying nuclear factor-kappaB expression. Nutr. Res. 28: 487-493.

Nunes, E.A., Lomax, A.R., Noakes, P.S., Miles, E.A., Fernandes, L.C., and Calder, P.C. 2011. beta-Hydroxy-beta-methylbutyrate modifies human peripheral blood mononuclear cell proliferation and cytokine production in vitro .Nutrition, 27: 92-99. Pimentel, G.D., Rosa, J.C., Lira, F.S., Zanchi, N.E., Ropelle, E.R., Oyama, L.M., Oller do Nascimento, C.M., de Mello, M.T., Tufik, S., and Santos, R.V. 2011. beta-Hydroxybeta-methylbutyrate (HMbeta) supplementation stimulates skeletal muscle hypertrophy in rats via the mTOR pathway. Nutr. Metab. (Lond.) 8: 11.

Pinheiro, C.H., Gerlinger-Romero, F., Guimaraes-Ferreira, L., de Souza-Jr, A.L., Vitzel, K.F., Nachbar, R.T., Nunes, M.T., and Curi, R. 2012. Metabolic and functional effects of beta-hydroxy-beta-methylbutyrate (HMB) supplementation in skeletal muscle. Eur. J. Appl. Physiol. 112: 2531-2537.

Pinheiro, C.H., Guiarães-Ferreira, L., Gerlinger-Romero, F. \& Curi, R. (2013). An Overview on Beta-hydroxy-beta-methylbutyrate) HMB) Supplementation in Skeletal Muscle Function and Sports Performance. In Nutrition and Enhanced Sports Performance. Edited by D.B.N. Sen. San Diego: Academic Press. pp. 455-463.

Reddy, S.S., Ramatholisamma, P., Karuna, R., and Saralakumari, D. 2009. Preventive effect of Tinospora cordifolia against high-fructose diet-induced insulin resistance and oxidative stress in male Wistar rats. Food Chem. Toxicol. 47: 2224-2229.

Seifter, S., Dayton, S., Novic, B., and Muntwyler, E. 1950. The estimation of glycogen with the anthrone reagent. Arch. Biochem. 25: 191-200.

Shawky, N.M., Shehatou, G.S., Abdel, R.M., Suddek, G.M., and Gameil, N.M. 2014. Levocetirizine ameliorates high fructose diet-induced insulin resistance, vascular dysfunction and hepatic steatosis in rats. Eur. J Pharmacol. 740: 353-363. 
Shiomi, Y., Yamauchi, T., Iwabu, M., Okada-Iwabu, M., Nakayama, R., Orikawa, Y., Yoshioka, Y., Tanaka, K., Ueki, K., and Kadowaki, T. 2015. A Novel Peroxisome Proliferator-activated Receptor (PPAR)alpha Agonist and PPARgamma Antagonist, Z551, Ameliorates High-fat Diet-induced Obesity and Metabolic Disorders in Mice. J. Biol. Chem. 290: 14567-14581.

Su, C.H., Liu, I.M., Chung, H.H., and Cheng, J.T. 2009. Activation of I2-imidazoline receptors by agmatine improved insulin sensitivity through two mechanisms in type-2 diabetic rats. Neurosci. Lett. 457: 125-128.

Su, K.H., Chandramouli, V., Ismail-Beigi, F., and Muzic, R.F., Jr. 2014.

Dexamethasone-induced insulin resistance: kinetic modeling using novel PET radiopharmaceutical 6-deoxy-6-[(18)F]fluoro-D-glucose. Mol. Imaging Biol. 16: 710720.

Takagawa, Y., Berger, M.E., Hori, M.T., Tuck, M.L., and Golub, M.S. 2001. Long-term fructose feeding impairs vascular relaxation in rat mesenteric arteries. Am. J. Hypertens. 14: $81.817-1$

Townsend, J.R., Hoffman, J.R., Gonzalez, A.M., Jajtner, A.R., Boone, C.H., Robinson, E.H., Mangine, G.T., Wells, A.J., Fragala, M.S., Fukuda, D.H., and Stout, J.R. 2015. Effects of beta-Hydroxy-beta-methylbutyrate Free Acid Ingestion and Resistance Exercise on the Acute Endocrine Response. Int. J. Endocrinol., 2015: doi: $10.1155 / 2015 / 856708$.

Van, K.M. and Nissen, S. 1992. Oxidation of leucine and alpha-ketoisocaproate to betahydroxy-beta-methylbutyrate in vivo. Am. J. Physiol. 262: E27-E31.

Vasudevan, H., Nagareddy, P.R., and McNeill, J.H. 2006. Gonadectomy prevents endothelial dysfunction in fructose-fed male rats, a factor contributing to the development of hypertension. Am. J. Physiol. 291: H3058-H3064. 
Virkamaki, A., Ueki, K ,.and Kahn, C.R. 1999. Protein-protein interaction in insulin signaling and the molecular mechanisms of insulin resistance. J. Clin. Invest. 103: 931943.

Wood, I.S. and Trayhurn, P. 2003. Glucose transporters (GLUT and SGLT): expanded families of sugar transport proteins. Br. J. Nutr. 89: 3-9.

Yadav, H., Jain, S., and Sinha, P.R. 2007. Antidiabetic effect of probiotic dahi containing Lactobacillus acidophilus and Lactobacillus casei in high fructose fed rats. Nutrition, 23: 62-68.

Yadav, H., Jain, S., Yadav ,M., Sinha, P.R., Prasad, G.B., and Marotta, F. 2009. Epigenomic derangement of hepatic glucose metabolism by feeding of high fructose diet and its prevention by Rosiglitazone in rats. Dig. Liver Dis. 41: 500-508. Ye, J. 2013. Mechanisms of insulin resistance in obesity. Front Med. 7: 14-24. Yonamine, C.Y., Teixeira, S.S., Campello, R.S., Gerlinger-Romero, F., Rodrigues, C.F., Jr., Guimaraes-Ferreira, L., Machado, U.F., and Nunes, M.T. 2014. Beta hydroxy beta methylbutyrate supplementation impairs peripheral insulin sensitivity in healthy sedentary Wistar rats. Acta. Physiol. (Oxf). 212: 62-74. 


\section{Figure legends}

Figure (1): Effect of HMB on HFD-induced changes in OGTT and ITT

Rats were fed 60\% HFD for 12 weeks and HMB (320 mg/kg/day, orally) was given from week 9 to 12 ; then OGTT (A) and ITT (B) were measured.

Data are expressed as mean $\pm \operatorname{SEM}(n=8)$.

* \# $p<0.05$, significantly different from control and HFD group respectively using one way ANOVA followed by Tukey-Kramer multiple comparisons post hoc test.

HMB: $\beta$-hydroxy- $\beta$-methylbutyrate; ANOVA: analysis of variance; AUC; area under the curve; HFD: high fructose diet; ITT: insulin tolerance test; OGTT: oral glucose tolerance test; SEM: standard error of mean.

Figure (2): Effect of HMB on HFD-induced changes in lipid profile in serum Rats were fed 60\% HFD for 12 weeks and HMB (320 mg/kg/day, orally) was given from week 9 to 12; then TC (A), TG (B), HDL-C (C), LDL-C (D) and VLDL-C (E) were measured.

Data are expressed as mean $\pm \operatorname{SEM}(n=8)$.

* , $p<0.05$, significantly different from control and HFD group respectively using one way ANOVA followed by Tukey-Kramer multiple comparisons post hoc test.

HMB: $\beta$-hydroxy- $\beta$-methylbutyrate; ANOVA: analysis of variance; HFD: high fructose diet; TC: total cholesterol; TG: triglycerides; HDL-C: high density lipoprotein cholesterol; LDL-C: low density lipoprotein cholesterol and VLDL-C: very low density lipoprotein cholesterol; SEM: standard error of mean.

Figure (3): Effect of HMB on HFD-induced changes in vascular reactivity in vitro Rats were fed $60 \%$ HFD for 12 weeks and HMB $(320 \mathrm{mg} / \mathrm{kg} /$ day, orally) was given from week 9 to 12 ; then aortic rings were isolated and tested for vascular reactivity by 
evaluating the contractile response to $80 \mathrm{mmol} / \mathrm{L}$ of $\mathrm{KCl}(\mathrm{A})$, vascular reactivity to cumulative concentration response curve of PE (B) and vascular relaxation to $\mathrm{ACh}(\mathrm{C})$ and SNP (D) after precontracting the aortic ring with PE; (E) a table representing $\mathrm{pEC}_{50}$ and $\mathrm{E}_{\max }$ values.

Data are expressed as mean $\pm \operatorname{SEM}(n=6)$.

* , $p<0.05$, significantly different from control and HFD group respectively using one way ANOVA followed by Tukey-Kramer multiple comparisons post hoc test.

ACh: acetylcholine; HMB: $\beta$-hydroxy- $\beta$-methylbutyrate; ANOVA: analysis of variance; HFD: high fructose diet; $\mathrm{KCl}$ : potassium chloride; PE: phenylephrine; ; SEM: standard error of mean; SNP: sodium nitroprusside; $\mathrm{E}_{\max }$ : maximum response and $\mathrm{pEC}_{50}$ : negative log of the concentration producing $50 \%$ of maximal response.

\section{Figure (4): Effect of HMB on HFD-induced changes in $m R N A$ expression of}

\section{GLUT-2, mTOR, SREBP-1c and PPAR- $\alpha$}

Rats were fed 60\% HFD for 12 weeks and HMB (320 mg/kg/day, orally) was given from week 9 to 12; then GLUT-2 (A), mTOR (B), SREBP-1c (C) and PPAR- $\alpha$ (D) were measured using real time RT-PCR.

Data are expressed as mean $\pm \operatorname{SEM}(n=6)$

* , $p<0.05$, significantly different from control and HFD group respectively using one way ANOVA followed by Tukey-Kramer multiple comparisons post hoc test.

HMB: $\beta$-hydroxy- $\beta$-methylbutyrate; ANOVA: analysis of variance; HFD: high fructose diet; GLUT-2: glucose transporter type-2; mTOR: the mammalian target of rapamycin; SREBP-1c: sterol regulatory element-binding protein-1 and PPAR- $\alpha$ : peroxisome proliferator-activated receptor-alpha; SEM: standard error of mean. 
Figure (5): Effect of HMB on HFD-induced changes in histopathological examination of liver

Rats were fed $60 \%$ HFD for 12 weeks and HMB (320 mg/kg/day, orally) was given from week 9 to 12 ; then liver specimen $(n=6)$ were collected and stained with H\&E. (A) Control $(H \& E \times 200)$ showing normal hepatocytes, $(B)$ HFD $(H \& E \times 200)$ showing severe steatosis, $(C)$ HMB $(H \& E \times 200)$ showing normal hepatocytes, (D) HFD+ HMB showing minimal steatosis $(H \& E \times 200)$ and $(E)$ figure showing scoring of steatosis.

Data are expressed as mean $\pm \operatorname{SEM}(n=6)$.

* , $p<0.05$, significantly different from control and HFD group respectively using Kruskal-Wallis followed by Dunn's multiple comparison test.

HMB: $\beta$-hydroxy- $\beta$-methylbutyrate; HFD: high fructose diet; SEM: standard error of mean. 


\section{Figure 1}

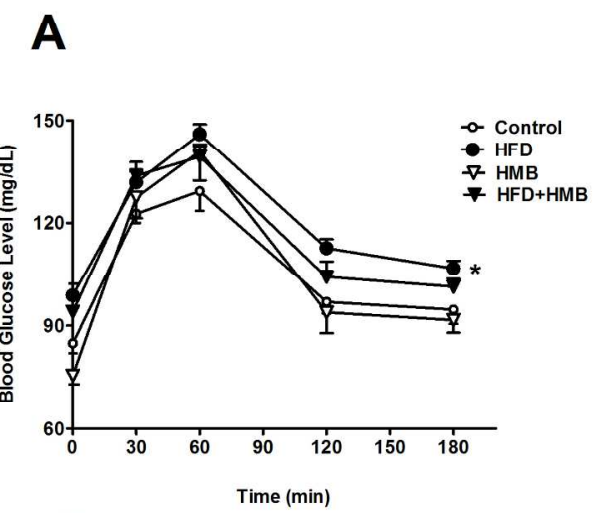

B

C

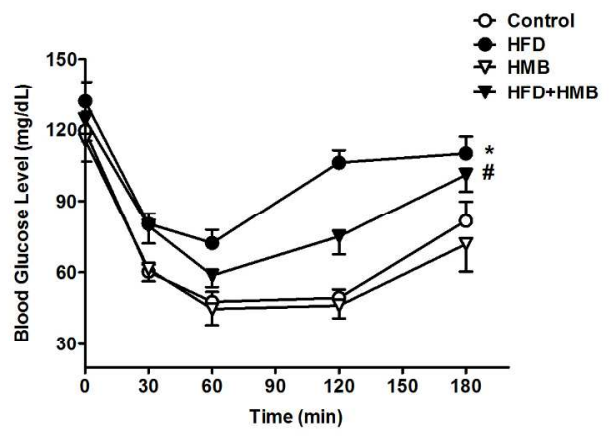

\begin{tabular}{|c|c|c|}
\hline Control & $\begin{array}{c}\text { AUC of Blood Glucose Level in } \\
\text { OGTT }\end{array}$ & $\begin{array}{c}\text { AUC of Blood Glucose Level in } \\
\text { ITT }\end{array}$ \\
\hline HFD & $19416.81 \pm 351.13$ & $10860.00 \pm 355.75$ \\
\hline HMB & $21959.67 \pm 343.14$ & $17316.67 \pm 773.09$ \\
\hline HFD+HMB & $20668.00 \pm 634.70$ & $10521 \pm 1061.40$ \\
\hline
\end{tabular}

Effect of HMB on HFD-induced changes in OGTT and ITT $200 \times 155 \mathrm{~mm}(300 \times 300 \mathrm{DPI})$ 
Figure 2
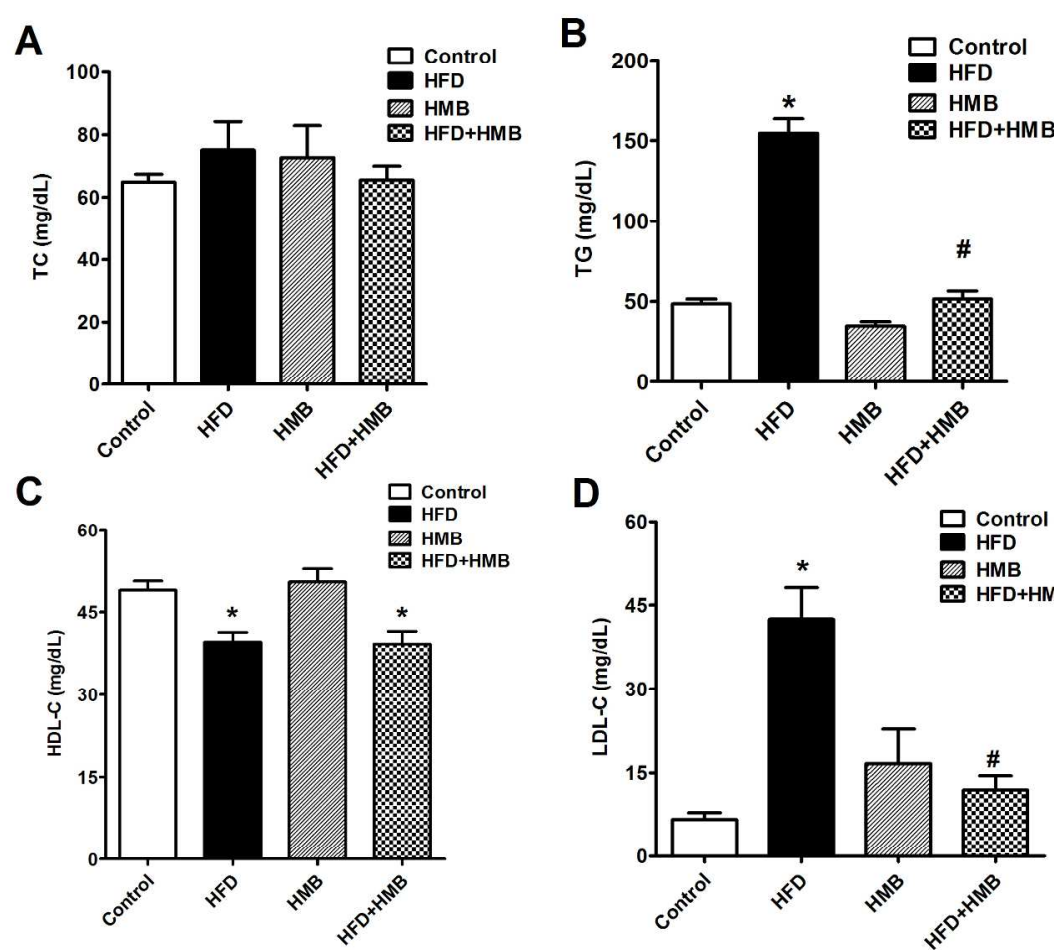

D

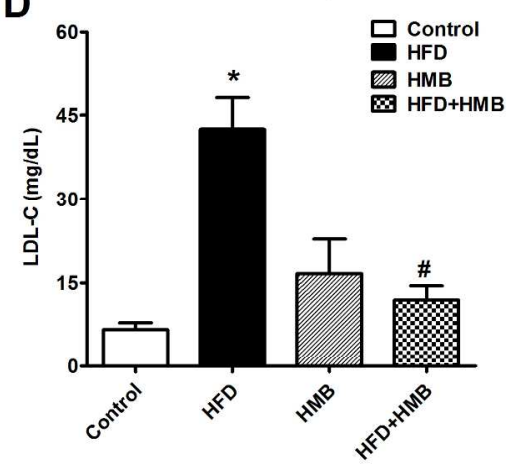

E

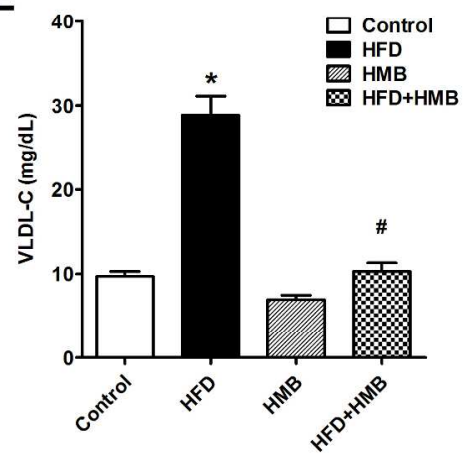

Effect of HMB on HFD-induced changes in lipid profile in serum $195 \times 273 \mathrm{~mm}$ (300 x $300 \mathrm{DPI})$ 
Figure 3

A

B
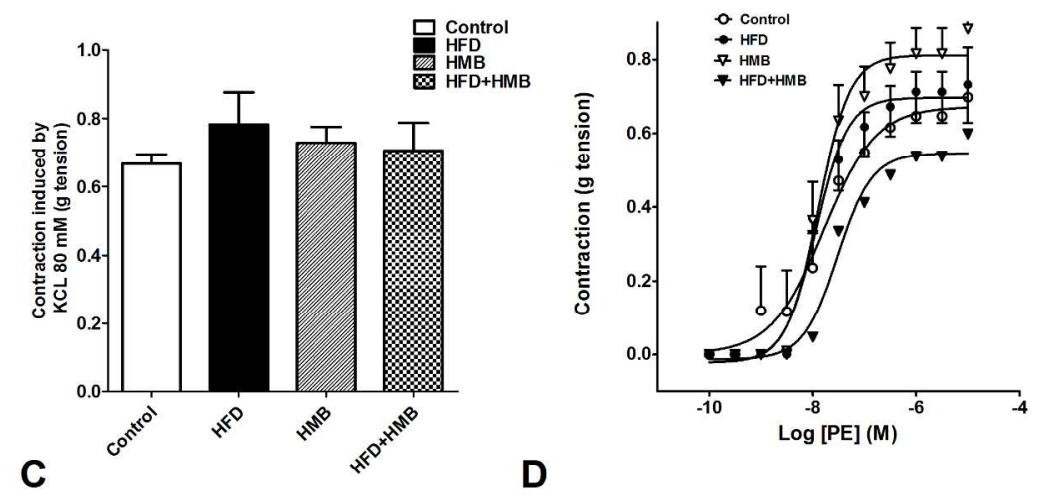

D
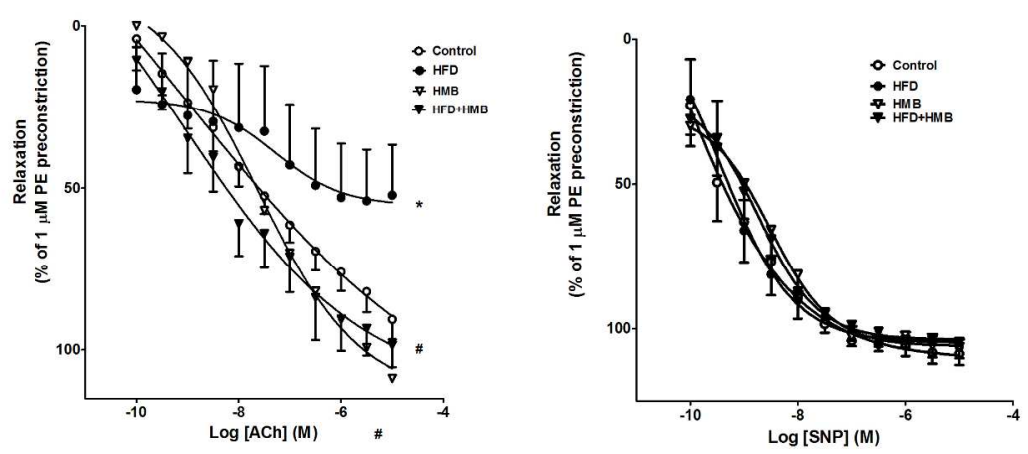

E

\begin{tabular}{|c|c|c|c|c|c|}
\hline \multicolumn{2}{|c|}{} & Control & HFD & HMB & HFD+HMB \\
\hline \multirow{2}{*}{ PE } & PEC $_{\mathbf{5 0}}$ & $7.63 \pm 0.25$ & $7.71 \pm 0.09$ & $7.49 \pm 0.35$ & $7.52 \pm 0.15$ \\
& $\mathbf{E}_{\max }$ & $0.65 \pm 0.06$ & $0.69 \pm 0.03$ & $0.62 \pm 0.08$ & $0.55 \pm 0.04$ \\
\hline \multirow{2}{*}{ Ach } & PEC $_{\mathbf{5 0}}$ & $8.99 \pm 0.77$ & $7.29 \pm 1.25$ & $7.64 \pm 0.18$ & $9.07 \pm 3.21$ \\
\cline { 2 - 6 } & $\mathbf{E}_{\max }$ & $127.90 \pm 7.22$ & $55.44^{*} \pm 4.37$ & $114.5 \pm 9.13$ & $113.8^{*} \pm 25.96$ \\
\hline \multirow{2}{*}{ SNP } & PEC $_{\mathbf{5 0}}$ & $8.89 \pm 0.18$ & $9.11 \pm 0.17$ & $8.43 \pm 0.14$ & $8.65 \pm 0.15$ \\
& $\mathbf{E}_{\max }$ & $104.80 \pm 3.17$ & $102.00 \pm 3.13$ & $104.4 \pm 2.69$ & $102.3 \pm 2.75$ \\
\hline
\end{tabular}

Figure (3): Effect of HMB on HFD-induced changes in vascular reactivity in vitro $200 \times 283 \mathrm{~mm}(300 \times 300 \mathrm{DPI})$ 
Figure 4

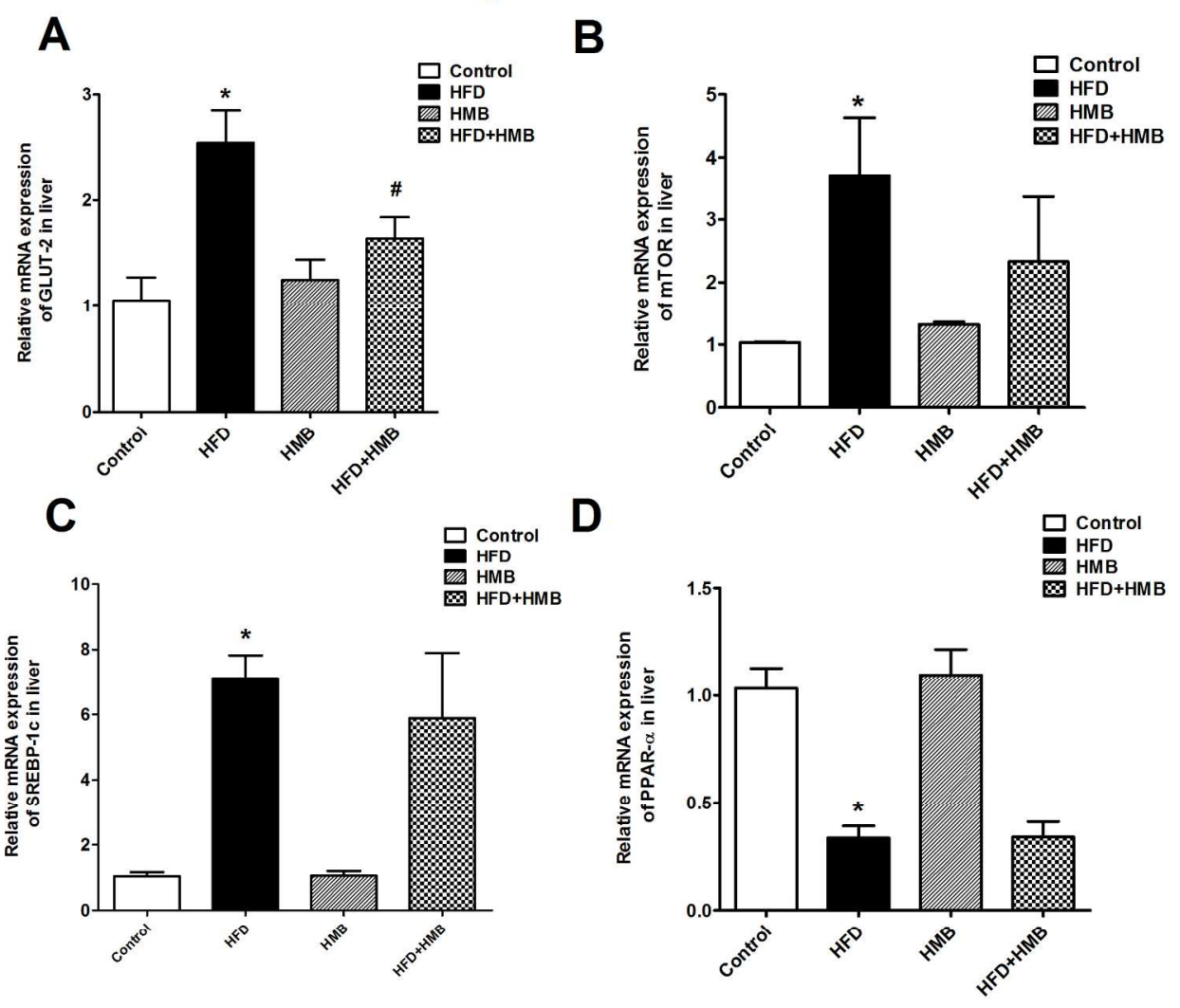

Figure (4): Effect of HMB on HFD-induced changes in mRNA expression of GLUT 2, mTOR, SREBP-1c and PPAR-a $205 \times 187 \mathrm{~mm}(300 \times 300 \mathrm{DPI})$ 
Figure 5
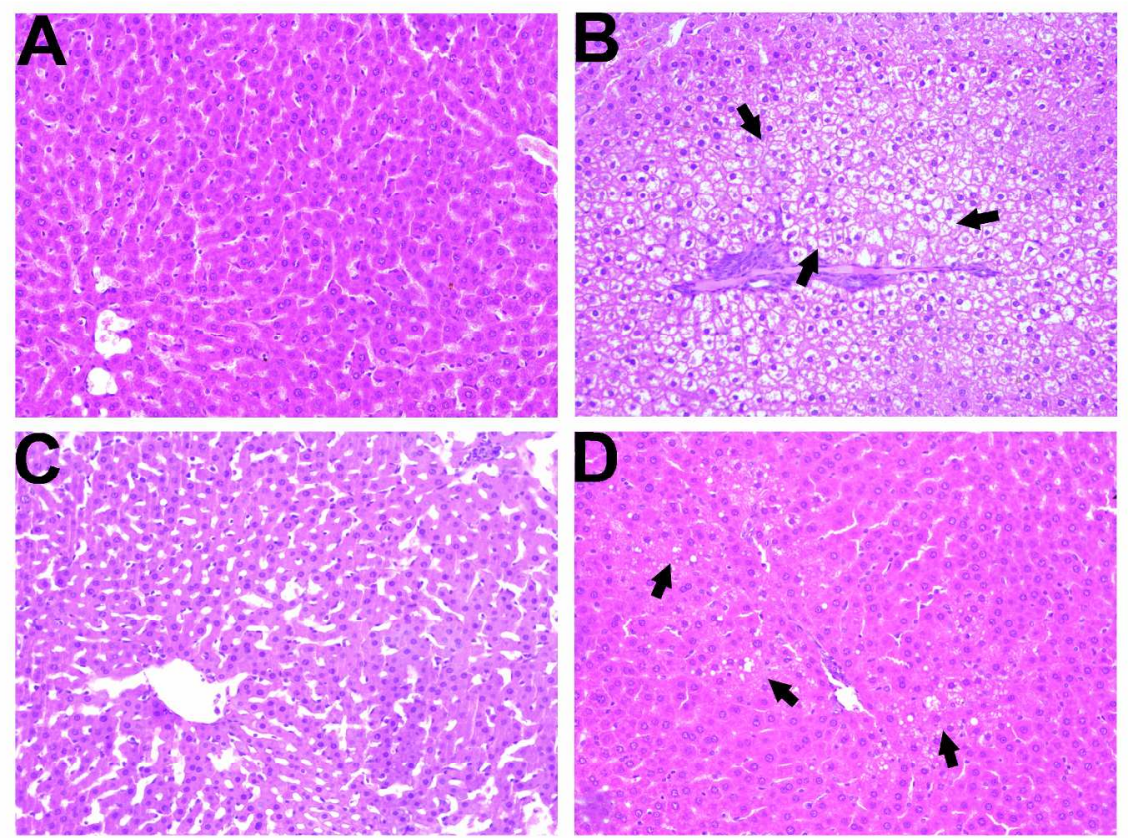

$\mathbf{E}$

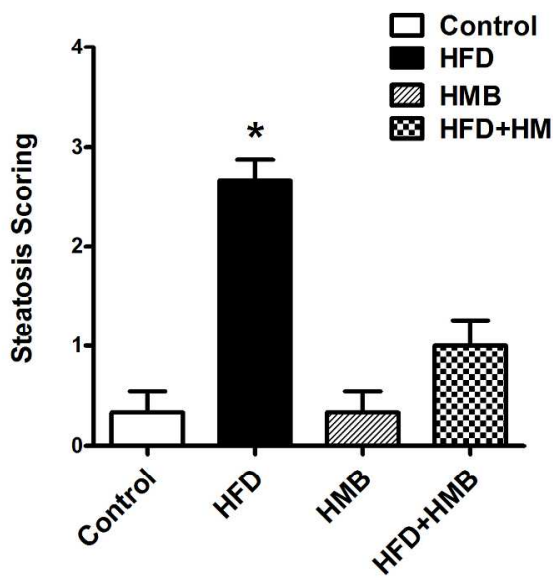

Figure (5): Effect of HMB on HFD-induced changes in histopathological examination of liver $201 \times 268 \mathrm{~mm}(300 \times 300$ DPI) 
Table (1): Effect of HMB on initial body weight, final body weight, body weight gain food intake, fasting serum glucose, insulin, HOMA-IR index, $\mathrm{Hb}_{1 c}$ and liver glycogen content

\begin{tabular}{|c|c|c|c|c||}
\hline & Control & HFD & HMB & HFD+HMB \\
\hline Initial body weight (g) & $141.63 \pm 2.15$ & $148.8 \pm 2.64$ & $132.50 \pm 2.18$ & $135.40 \pm 1.76$ \\
\hline Final body weight (g) & $315.88 \pm 13.61$ & $308.5 \pm 12.76$ & $271.40 \pm 15.84$ & $286.60 \pm 10.16$ \\
\hline Body weight gain (g) & $174.25 \pm 13.75$ & $159.7 \pm 13.59$ & $130.40 \pm 11.02$ & $151.20 \pm 10.38$ \\
\hline Food intake (g/day/rat) & $33.32 \pm 1.21$ & $30.24 \pm 1.65$ & $33.02 \pm 1.25$ & $32.44 \pm 1.02$ \\
\hline Fasting Insulin ( $\mu$ g/L) & $0.43 \pm 0.06$ & $1.49^{*} \pm 0.09$ & $0.68^{\#} \pm 0.06$ & $1.21^{* \#} \pm 0.04$ \\
\hline Fasting Glucose (mg/dl) & $111.46 \pm 3.05$ & $121.39^{*} \pm 1.72$ & $107.92 \pm 2.38$ & $115.08 \pm 2.37$ \\
\hline HOMA-IR index & $1.39 \pm 0.12$ & $5.03^{*} \pm 0.24$ & $1.07 \pm 0.09$ & $3.67^{* \#} \pm 0.18$ \\
\hline HbA $(\%)$ & $4.17 \pm 0.22$ & $5.73^{*} \pm 0.34$ & $4.65 \pm 0.12$ & $4.17^{\#} \pm 0.66$ \\
\hline Glycogen (mg/100g tissue) & $484.44 \pm 56.15$ & $1004.99^{*} \pm 127.51$ & $509.43 \pm 59.72$ & $576.55^{\#} \pm 79.20$ \\
\hline
\end{tabular}

Rats were fed 60\% HFD for 12 weeks and HMB (320 mg/kg/day, orally) was given from week 9 to 12 ; then fasting glucose, insulin, $\mathrm{HOMA}-\mathrm{IR}$ index, $\mathrm{HbA}_{1 \mathrm{c}}$ and liver glycogen content were measured. The body weight was measured weekly and food intake was estimated daily.

Data are expressed as mean $\pm \operatorname{SEM}(n=8)$.

*, \# $p<0.05$, significantly different from control and HFD group respectively using one way ANOVA followed by Tukey-Kramer multiple comparisons post hoc test.

HMB: $\beta$-hydroxy- $\beta$-methylbutyrate; ANOVA: analysis of variance; HFD: high fructose diet; $\mathrm{HbA}_{1 \mathrm{c}}$ : Glycosylated hemoglobin; HOMA-IR index: homeostasis model assessment of insulin resistance index; SEM: standard error of mean. 